hep-lat/9910019

\title{
Results and Perspectives in HEP, vis-a-vis Lattice QCD
}

\author{
M.L. Mangano ${ }^{\mathrm{a} *}$ \\ ${ }^{a}$ CERN, Theoretical Phisics Division 1211 Geneva 23, Switzerland
}

I review in this presentation some aspects of phenomenology in High Energy Physics which are related to recent and possibly future progress in lattice QCD. In particular, I cover (i) the extraction of CKM matrix elements from $B$ physics, (ii) the determination of $\epsilon^{\prime} / \epsilon$, as well as (iii) some issues emerged in the physics of high energy jets produced in hadronic collisions, where input from non-perturbative calculations would benefit our capability to perform better theoretical predictions.

\section{INTRODUCTION}

The last couple of years have given us an impressive series of important new results in HEP. Most of them, if not all, are in the field of flavour physics. We obtained conclusive evidence for neutrino mixing and therefore for neutrino masses (SuperKamiokande); new results on $\epsilon^{\prime} / \epsilon$ (KTeV, NA48); the first strong evidence for CP violation in the $B$ system (CDF); the first direct limits on $B_{s}$ mixing (LEP, SLC). Impressive progress is thus taking place in the area of flavour physics, and these results are just the appetizer for much more to come in the next few years. New data are in fact soon expected from the Bfactories (BaBar and Belle, both of which successfully started in the Summer their operations, and CLEO), from the upcoming run of the Tevatron (CDF, D0), from the analyses of the full datasets of $\mathrm{KTeV}$ and NA48, from the first operation of the KLOE experiment at the Daphne $\phi$ factory, and from the new long-baseline neutrino beam from KEK to SuperKamiokande. Finally, a series of new experiments has recently been (or hopefully will soon be) approved: searches for rare kaon decays (FNAL, BNL), searches for $\mu \rightarrow e \gamma$ decays (KEK, PSI) and new long baseline neutrino-

\footnotetext{
*This work was supported in part by the EU Fourth Framework Programme "Training and Mobility of Researchers", Network "Quantum Chromodynamics and the Deep Structure of Elementary Particles", contract FMRX-CT98-0194 (DG 12 - MIHT).
}

oscillation experiments (MINOS at FNAL, NGS at CERN/Gran Sasso).

On the theoretical side, the problem of flavour is among the most interesting ones. Flavour physics provides a key peephole to explore and possibly uncover the existence of new phenomena beyond the Standard Model. In supersymmetry, the problem of flavour is receiving nowadays as much attention as the problem of SUSY breaking, if not more, and the two problems are universally believed to be intimately related. The observation of flavour phenomena not explained within the SM would provide valuable input for the understanding of physics at the SUSY breaking scale.

For the experimental information to be useful in this direction, it is of fundamental importance that accurate data be compared with accurate SM predictions. With the exception of the observation of neutrino masses, the interpretation of all the new input on flavour physics coming from the observations listed above heavily relies on non-perturbative physics, which lattice calculations are best suited to carry out. Almost anything being calculated on the lattice is fundamental to interpret the available and forthcoming data: the efforts of the lattice community are therefore much appreciated by all of us living in the continuum!

I will review in this talk the status of the experimental inputs, and the perspectives for future improvements. As an outsider in the field of lat- 
tice, I hope this review will be useful to those of you mostly involved in the numerical aspects of lattice physics. I apologise to those of you who are experts in the phenomenological aspects I will discuss, and who may find this review incomplete, or too naive. Hopefully the bibliography will help filling in some gaps. I will concentrate on the following selection of topics: the status of the extraction of the CKM matrix elements from $B$ physics, and the measurement of $\epsilon^{\prime} / \epsilon$. In both cases, I will make heavy use for this presentation of very good, recent extensive reviews of the subjects. In addition, I will cover a couple of topics taken from current high-energy phenomenology: prompt-photon production in fixed target, and production of high-energy jets in hadronic collisions. Evidence is emerging that a better understanding of the interface between the perturbative and non-perturbative domain is required to solve some problems in this area. Whether the lattice approach can provide some useful input to address these issues, it is too early to say, and hard for me to judge. I hope the following discussion will anyway serve as a stimulus for some of you to try address these questions.

\section{CKM PARAMETERS FROM B PHYSICS}

$V_{\mathrm{CKM}}$ is the matrix describing the couplings of the weak charged currents of quarks to the $W$ gauge boson:

$L_{E W K} \sim V_{i j} \bar{Q}^{i} \gamma_{\mu}\left(1-\gamma_{5}\right) Q^{j} W^{\mu}$

With 3 generations, the CKM matrix can be parameterised as follows:

$$
\begin{aligned}
& \left(\begin{array}{ccc}
V_{u d} & V_{u s} & V_{u b} \\
V_{c d} & V_{c s} & V_{c b} \\
V_{t d} & V_{t s} & V_{t b}
\end{array}\right)= \\
& \left(\begin{array}{ccc}
1-\frac{\lambda^{2}}{2} & \lambda & A \lambda^{3}(\rho-i \eta) \\
-\lambda & 1-\frac{\lambda^{2}}{2} & A \lambda^{2} \\
A \lambda^{3}(1-\rho-i \eta) & -A \lambda^{2} & 1
\end{array}\right)
\end{aligned}
$$

Here $\lambda=\sin \theta_{c}$ is the sine of the Cabibbo angle, and we neglected $\mathcal{O}\left(\lambda^{4}\right)$ corrections. In addition to the Cabibbo angle, 3 more parameters are required to parametrize the matrix: 2 real numbers, and a phase. A direct determination of the $V_{\mathrm{CKM}}$ elements [1] should be obtained from tree-level processes, to avoid possible contaminations from physics beyond the SM. Unfortunately, this is not possible for all entries: no direct measurement of $V_{t d, t s}$ has been possible so far, and a crude measurement of $V_{t b} \sim 1$ is only possible if one assumes knowledge of the $t \bar{t}$ production cross section at the Tevatron. The comparison with observables induced by higher-order processes (e.g. $K^{0}-\bar{K}^{0}$ mixing, $\left.\propto\left(V_{c s} V_{c d}^{*}\right)^{2}\right)$, provides consistency checks, potential measurements of relative phases, and possible information on BSM phenomena.

\section{1. $\left|\mathrm{V}_{\mathrm{cb}}\right|$}

$\left|V_{c b}\right|$ is measured from both exclusive and inclusive semileptonic decays of $B$ mesons with charmed hadrons in the final state. Since these processes proceed at tree level, the determination of $\left|V_{c b}\right|$ is with very good accuracy free of possible contaminations from new physics.

The inclusive extraction is obtained from the measurement of the inclusive lifetime, and of the semileptonic branching ratio (B). The theoretical estimates of lifetime (for reviews, see e.g. ref. [2]) have uncertainties from the knowledge of the $b$ and $c$ quark masses, of the heavy quark kinetic energy inside the hadron, and of higher-order corrections to the perturbative expansions in powers of $\alpha_{S}$ (known to NNLO), and in powers of $1 / m_{Q}$ (known to $\mathcal{O}\left(1 / m_{Q}^{2}\right)$ ). The contribution of these sources of uncertainties to the determination of $\left|V_{c b}\right|$ is given by the following formula (uncertainties form the recent compilation in ref. (3)):

$$
\begin{array}{r}
\left|V_{c b}\right|=0.0411 \sqrt{\frac{B\left(B \rightarrow \ell \nu X_{c}\right)}{0.105}} \sqrt{\frac{1.55 \mathrm{ps}}{\tau(B)}} \times \\
{\left[1-0.024 \frac{\mu_{\pi}^{2}-0.5}{0.1 \mathrm{GeV}^{2}}\right] \times} \\
{\left[1 \pm 0.030_{\mid P T} \pm 0.020_{\mid \Delta m_{b}} \pm 0.024_{\mid 1 / m_{Q}^{3}}\right]}
\end{array}
$$

The term proportional to $\mu_{\pi}^{2}$ parametrizes the quark kinetic energy, and reflects the uncertainty in the extraction of the $b-c$ mass difference from 
the HQET relation [2]3]:

$$
\begin{aligned}
m_{b}-m_{c} & =\left\langle M_{B}\right\rangle-\left\langle M_{D}\right\rangle+\mu_{\pi}^{2}\left(\frac{1}{2 m_{c}}-\frac{1}{2 m_{b}}\right) \\
& +\mathcal{O}\left(1 / m_{c, b}^{2}\right) \\
& \sim 3.50 \mathrm{GeV}+40 \mathrm{MeV} \cdot \frac{\mu_{\pi}^{2}-0.5}{0.1 \mathrm{GeV}^{2}}
\end{aligned}
$$

The latest results on the inclusive extraction of $\left|V_{c b}\right|$ come from the average of the LEP measurements [四:

$\left|V_{c b}\right|=\left(40.75 \pm 0.41_{\exp } \pm 2.04_{t h}\right) \times 10^{-3}$

Notice that the theoretical error is much larger than the current experimental uncertainties.

The exclusive extraction requires the theoretical knowledge of the form factor for $B \rightarrow D^{*} \ell \nu$ at zero recoil. Spin symmetry in the $m \rightarrow \infty$ limit gives $F(1)=1$ [2]. Finite mass corrections are evaluated within HQET, giving (for a recent review see |5]; this value was taken from the compilation in ref. [3]) $F_{D^{*}}(1)=0.88 \pm 0.08$, which is consistent with the most recent determinations from the lattice presented at this Conference [6], $F_{D^{*}}(1)=0.935 \pm 0.035$. The current average of the exclusive LEP results gives [4]:

$\left|V_{c b}^{\text {excl }}\right|=\left(38.4 \pm 2.5_{\exp } \pm 2.2_{\mathrm{th}}\right) \cdot 10^{-3}$

and the world average, including CLEO, is [7]:

$\left|V_{c b}^{e x c l}\right| \times 10^{4}=385 \pm 9_{s t} \pm 16_{\text {syst }} \pm 26_{t h}$,

which includes the correlations of experimental and theoretical uncertainties of the various experimental results.

The current world average of the CLEO and LEP inclusive and exclusive results [7] is:

$\left|V_{c b}\right| \times 10^{4}=400 \pm 4_{e x p} \pm 21_{t h}$.

Again, notice the large gap in accuracy between the experimental and the theoretical precisions.

\section{2. $\left|\mathrm{V}_{\mathrm{ub}}\right|$}

The measurement of $\left|V_{u b} / V_{c b}\right|=|\lambda(\rho-i \eta)|$ provides the first direct constraint on the $(\rho, \eta)$ plane, in the form of a circle centered around the origin. $\left|V_{u b}\right|$ is measured in charm-less $B$ decays. In particular, the used observables are:
- exclusive decays such as $B \rightarrow \pi \ell \nu, B \rightarrow \rho \ell \nu$;

- the end-point spectrum in inclusive $B \rightarrow \ell X$ decays;

- low-mass hadronic recoils $X_{u}$ in $B \rightarrow \ell X_{u}$

Each one of these tecniques has different sets of theoretical and experimental systematics, which limit the potential accuracy of the measurement. For example, measurements based on the study of the end-point lepton spectrum rely on the assumed knowledge of the lepton spectrum away fom the end-point, in the low-energy region where production is dominated by charmed decays. The measurement done requiring a low-mass hadronic recoil system can be affected by the assumptions made on the exclusive structure of the final state, since these enter in the experimental definition of the sample itself.

Exclusive decays are experimentally much cleaner, but the theoretical interpretation is based on the assumed knowledge of the exclusive form factors for heavy-to-light transitions. The application of HQET is not reliable, since the $b$ decays to a light quark. One therefore has to rely on phenomenological models, or on lattice calculations. These, however, are less trustable for $B \rightarrow \pi \ell \nu$ than for $B \rightarrow \rho \ell \nu$ transitions. Current error estimates on the exclusive form factors are of the order of $\pm 15 \%$. Some new results have been presented at this conference, and were reviewed in the plenary talk by Hashimoto [8].

The latest measurements from CLEO [9] rely on $B \rightarrow \rho \ell \nu$ exclusive decays, and the result is:

$10^{3} \cdot\left|V_{u b}\right|=3.25 \pm 0.14_{\text {stat }}+0.21 \quad \pm 0.29_{\text {syst }} \pm 0.55_{\text {th }}(10)$

In the case of inclusive decays, calculations rely on the OPE and HQET. Theoretically, the main uncertainties come from higher-order PT corrections, and from $m_{b}$, since the phase-space is proportional to $m_{b}^{5}$. The relation between $\left|V_{u b}\right|$ and $B\left(B \rightarrow \ell \nu X_{u}\right)$, which is currently used by the LEP experiments, is given by the following expression [10]

$$
\begin{aligned}
\left|V_{u b}^{i n c l}\right|= & 0.00445 \sqrt{\frac{B\left(B \rightarrow \ell \nu X_{u}\right)}{0.002}} \sqrt{\frac{1.55 \mathrm{ps}}{\tau(B)}} \times \\
& \left(1 \pm 0.020_{P T} \pm 0.035_{\delta m_{b}}=60 \mathrm{MeV}\right)(11)
\end{aligned}
$$


The $60 \mathrm{MeV}$ uncertainty on $m_{b}\left(m_{b}(1 \mathrm{GeV})=\right.$ $4.58 \pm 0.06)$, although perhaps a bit optimistic, is consistent with the most recent estimates based on Sum Rules and NNLO QCD studies of the $\Upsilon$ spectrum [1], as well as with the most recent unquenched lattice estimates [12].

It should be pointed out that even if it were possible to calculate with high accuracy the full, charm-less inclusive rate, large uncertainties would still remain in the extraction of $B\left(B \rightarrow \ell \nu X_{u}\right)$ from the data. This is because the phase-space region used by experiments is only a very small fraction of the total one, in order to suppress the large $b \rightarrow c X$ backgrounds. For this reason, the measurement of $B\left(B \rightarrow \ell \nu X_{u}\right)$ is also affected by uncertainties in the modeling of the structure of the final states, which are in principle of theoretical origin. The most recent LEP results give 13]:

$$
\begin{aligned}
& 10^{3} \times B\left(B \rightarrow \ell \nu X_{u}\right)= \\
& 1.67 \pm 0.35_{\exp } \pm 0.38_{b} \rightarrow c \pm 0.20_{b} \rightarrow u
\end{aligned}
$$

where the last two errors come from the modeling systematics. Using this result and eq. (11), LEP obtains:

$\left|V_{u b}\right|=\left(\begin{array}{c}4.05_{-0.74}^{+0.62} \\ -0.3\end{array} 0^{-3}\right.$

where the overall error is approximately $40 \%$ experimental and $60 \%$ theoretical.

NLO corrections to the extraction of $V_{u b}$ using low-mass $X_{u}$ have recently been calculated 14. An estimate of the residual systematic uncertainty in the range of $\sim 10 \%$ was quoted.

It is important to point out that the accuracy of future data from the B factories will be dominated by exclusive decays. For example, the studies presented in the BaBar Physics Book [15] anticipate $\delta_{\text {exp }}^{\text {excl }} \sim 2.5 \%$, with $\delta_{\text {exp }}^{\text {incl }} \sim 15 \%$. One can presumably conclude that efforts for an improved theoretical determination of $V_{u b}$ should concentrate on the calculation of exclusive form factors, where lattice QCD can play a major role.

\section{3. $\left|\mathbf{V}_{\text {td }}\right|$}

The remaining measurements/constraints on $V_{\text {CKM }}$ require loop-level processes, since they all involve couplings of the top quark to the $s$ and $d$ quarks, none of which can be measured today directly in top decays. To interpret these measurements, one must therefore assume the SM. Massive BSM particles could in fact propagate within the loops, and spoil the connection to the $V_{\mathrm{CKM}}$ entries. Within the SM we have $V_{t s}=-V_{c b}$, and the last independent entry is therefore $\left|V_{t d}\right|$. Its less indirect measurement comes from the mixing of $B^{0}-\bar{B}^{0}$, mediated by box diagrams similar to those occurring in $K^{0}-\bar{K}^{0}$ mixing:

$\Delta m_{B_{d}} \propto\left|V_{t d} V_{t b}^{*}\right|^{2} f_{B_{d}}^{2} B_{B_{d}}$

Examples of BSM contributions to this process are given, for example, by SUSY boxes, where stop and charginos can propagate in the loop replacing top and $W$ 's.

The current value of $\left|V_{t d}\right|$, as obtained from the official average of the LEP, SLD and CDF results, is given by [16]:

$$
\begin{aligned}
\Delta m_{B_{d}} & =0.481 \pm 0.017 \mathrm{ps}^{-1} \\
\left|V_{t d}\right| & =(8.4 \pm 1.6) \times 10^{-3}
\end{aligned}
$$

$\left|V_{t d}\right|$ gives an independent constraint on $(\rho, \eta)$, represented by a circle centered around the point $\rho=1$. Perfect knowledge of $\left|V_{t d}\right|$ and of $\left|V_{u b}\right|$, therefore, would give unambiguous evidence ofCP in the CKM model, in spite of the fact that neither of the two observables is by itself $\mathrm{CP}$ violating.

In practice, the extraction of $\left|V_{t d}\right|$ from $\Delta m_{B_{d}}$ is limited theoretically by the imprecise 17 knowledge of

$f_{B_{d}} \sqrt{B_{B_{d}}}=210 \pm 40 \mathrm{MeV}$

Notice that the experimental accuracy is at the level of $3 \%$, and will significantly improve over the next few years, using the results from the Tevatron and the $\mathrm{B}$ factories. Will lattice calculations ever match this accuracy? Progress will hopefully come form the use of the experimentally determined values of $f_{D_{s}}$ and $f_{D}$ (extracted from $D \rightarrow \ell \nu$ decays), together with lattice QCD estimates of $f_{D} / f_{B_{d}}$ and $f_{D_{s}} / f_{D}$. One hopes to reach an accuracy of $5 \%$ on $B_{B}$ (lattice QCD) and of $5 \%$ on $f_{B_{d}}$, with a total uncertainty of 5-10 MeV on $f_{B_{d}} \sqrt{B_{B_{d}}}$. The impact of these improvements is discussed in [18]. 
2.4. $\Delta \mathrm{m}_{\mathrm{s}}$ and $\left|\mathrm{V}_{\mathrm{ts}} / \mathrm{V}_{\mathrm{td}}\right|$

A large fraction of the theoretical uncertainties on $\left|V_{t d}\right|$ disappears in the ratio:

$\frac{\Delta m_{s}}{\Delta m_{d}}=\frac{m_{B_{s}}}{m_{B_{d}}}\left|\frac{V_{t s}}{V_{t d}}\right|^{2} \xi^{2}$

with

$\xi=\frac{f_{B_{s}} \sqrt{B_{B_{s}}}}{f_{B_{d}} \sqrt{B_{B_{d}}}}=1.11 \pm 0.13$

with the error dominated by $f_{B_{s}} / f_{B_{d}}$, since $B_{B_{s}} / B_{B_{d}}=1.01 \pm 0.01$ [19].

$B_{s}$ oscillations have not been observed as yet. The current 95\% CL limit from LEP/SLD is [16]:

$\Delta m_{s}>14.3 \mathrm{ps}^{-1} \quad\left(x_{s} \gtrsim 21\right)$

leading to:

$\left|V_{t d}\right| /\left|V_{t s}\right|<0.24 \Rightarrow\left|V_{t d}\right|<0.010$

At small $\rho$, this constraint is as strong as the measurement of $\left|V_{t d}\right|$ !

\subsection{Global fits in the $(\rho, \eta)$ plane}

The set of measurements discussed above has been analysed recently by several groups [18], 20-23 which find evidence, at better than $95 \% \mathrm{CL}$, for $\eta>0$. I recall here the results of the study by Parodi et al. (PRS) 18]. The countour lines of their fits are shown in fig. 1. Evidence for

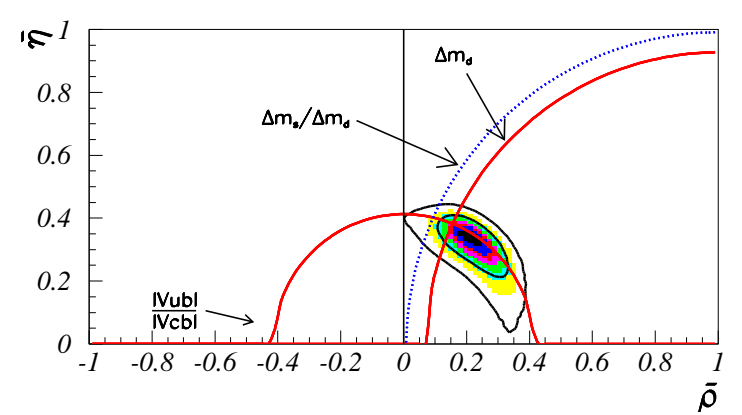

Figure 1. Confidence-level countour plots, form global fits to the $\rho$ and $\eta$ parameters using $B$ decay observables (from ref. [18]).

$\eta>0$ is even stronger if information from the $\epsilon$ parameter in $K^{0} \bar{K}^{0}$ mixing is used (see [18]).
Removing the various inputs for the fit one at the time, PRS obtain the results shown in table 1 Notice that:

- $B_{K}>0.60$ at $98.4 \% \mathrm{CL}$

- The current accuracy on $\sin 2 \beta$, obtained from the overall fit of non CP-violating observables, is similar to that expected after $\sim 3-4$ yrs of running at a B-factory! The fitted value of $\sin 2 \beta$ is consistent at this time with the CDF measurement 24.

- $f_{B_{d}} \sqrt{B_{B_{d}}}$, as determined from the global fit, has a better accuracy than what can be achieved today from lattice QCD!

- The best fit value for $x_{s}$ suggests that the observation of $B_{s}$ mixing is behind the corner. While LEP and SLD may not be able to see it, having almost completed their data analysis, CDF should have no problem to detect it during its forthcoming new run.

In view of the above results, and of the estimates for the performance expected from the upcoming experiments, it is interesting to ask how far can the theoretical accuracy be pushed, and what would one gain from this improved accuracy. Table 2 contains a summary of the theoretical uncertainties, and exptected experimental ones (1-3 yrs of B-factory, from the BaBar book [15]).

Some comments:

- Once $\Delta m_{s}$ will be measured, the ratio $\Delta m_{s} / \Delta m_{d}$ will provide the best determination of $|1-(\rho+i \eta)|$ (with the SM assumption)

- This determination could also be less affected by possible contaminations from new physics. For example, $\operatorname{MSSM}\left(\tilde{t}, \tilde{\chi}^{ \pm}\right)$boxes would cancel in the ratio

- $\Delta m_{d}$ can then be used to explore possible deviations from the SM. Assuming the SM relation $\left|V_{t d}\right|=\left|V_{t d} V_{c b} / V_{t s}\right|$, the reach for these explorations will be by and large determined by the uncertainty in $f_{B_{d}} \sqrt{B_{B}}$, until this reaches an accuracy comparable to $\left|V_{c b}\right|$. 
Table 1

Values of the parameters and inputs used in the fits to the $B$-decay observables described above, in addition to $\epsilon_{K}$. Results and table taken from ref. [18]. The two values given for $V_{u b} / V_{c b}$ correspond to the CLEO and LEP detemrinations, respectively.

\begin{tabular}{ccc}
\hline Parameter & Fitted value & Present value \\
\hline$\Delta m_{s}$ & $(14.8 \pm 2.8) p s^{-1}$ & $>12.3 \mathrm{ps}^{-1}$ at $95 \% \mathrm{CL}$ \\
$\left|\frac{V_{u b}}{V_{c b}}\right|$ & $0.097_{-0.022}^{+0.033}$ & $0.080 \pm 0.017 / 0.104 \pm 0.019$ \\
$B_{K}$ & $0.87_{-0.20}^{+0.34}$ & $0.86 \pm 0.09$ \\
$f_{B_{d}} \sqrt{B_{B_{d}}}$ & $(233 \pm 13) \mathrm{MeV}$ & $\left(210_{-32}^{+39}\right) \mathrm{MeV}$ \\
$m_{t}\left(m_{t}\right)$ & $\left(179_{-34}^{52}\right) \mathrm{GeV}$ & $(167 \pm 5) \mathrm{GeV}$ \\
$\left|V_{c b}\right|$ & $\left(42_{-4.0}^{+8.0}\right) \times 10^{-3}$ & $(40.0 \pm 2.2) \times 10^{-3}$ \\
$\sin 2 \beta$ & $0.725_{-0.060}^{+0.050}$ & $0.79_{-0.44}^{+0.41}(\mathrm{CDF}[24])$ \\
$\sin 2 \alpha$ & $-0.26_{-0.28}^{+0.29}$ & - \\
$\gamma$ & $\left(59.5_{-7.5}^{+8.5}\right)^{\circ}$ & - \\
\hline
\end{tabular}

Table 2

Current theoretical uncertainties, and projected experimental uncertainties, for $3^{\text {rd }}$ generation entries of the CKM matrix.

\begin{tabular}{lcll}
\hline Quantity & Theory unc. & Main source & Projected experimental unc. \\
\hline$\left|V_{c b}^{\text {incl }}\right|$ & $\pm 5 \%$ & $m_{Q}, \mathrm{PT}, \mathcal{O}\left(1 / m_{Q}^{3}\right)$ & $<1 \%$ \\
$\left|V_{c b}^{\text {excl }}\right|$ & $\pm 5 \%$ & $F_{D^{*}}(1): \mathcal{O}\left(1 / m_{Q}^{3}\right), D^{* *}$ & $1.5 \%$ \\
$\left|V_{u b}^{\text {incl }}\right|$ & $\pm(4-10) \%$ & $m_{b}, \mathrm{PT}$ & $\sim 15 \%$ \\
$\mid V_{u b}^{\text {excl }}$ & $\pm 15 \%$ & $\mathrm{FF}$ & $\sim 2.5 \%$ \\
$\left|\left(V_{u b} / V_{c b}\right)^{\text {incl }}\right|$ & $<3 \%$ & $m_{Q}$ & $?$ \\
$\left|V_{t d}\right|$ & $\pm 20 \%$ & $f_{B_{d}} \sqrt{B_{B}}$ & $\delta\left(\Delta m_{d}\right) \sim 1 \%_{s t a t} \oplus 1.5 \%_{\Gamma^{0}}$ \\
$\left|V_{t s} / V_{t d}\right|$ & $\pm 5 \% ?$ & $f_{B_{d}} / f_{B_{s}}$ & $\mathrm{CDF}(\mathrm{Run} \mathrm{II}): \delta\left(\Delta m_{s}\right) \sim 2 \%$ \\
\hline
\end{tabular}

As an example of the impact of improved accuracies in $f_{B_{d}} \sqrt{B_{B}}$ for searches of new physics effects, consider the constraints on $m(\tilde{t}), m\left(\tilde{\chi}^{ \pm}\right)$ from $B_{d}$ mixing. For a higgsino-like chargino, the supersymmetric box contribution to $B_{d}$ mixing [25] is given by:

$\frac{\left(\Delta m_{d}\right)_{S U S Y}}{\left(\Delta m_{d}\right)_{S M}} \simeq\left(\frac{m_{t}}{m_{\tilde{\chi}}}\right)^{2} \frac{1}{2.2 \sin ^{4} \beta} G\left(\frac{m_{\tilde{t}}}{m_{\tilde{\chi}}}\right)$.

The function $G(x)$ is a slowly varying function of $x$, given explicitly in ref. [25], which takes the value $G(1)=\frac{1}{3}$. In the most conservative case of large $\tan \beta(\sin \beta=1)$, we get the following effects:

\begin{tabular}{lcccc}
\hline$m_{\tilde{\chi}^{ \pm}}=m_{\tilde{t}}(\mathrm{GeV})$ & 100 & 150 & 200 & 300 \\
$\left(\Delta m_{d}\right)_{S S} /\left(\Delta m_{d}\right)_{S M}$ & 0.46 & 0.21 & 0.12 & 0.05 \\
\hline
\end{tabular}

No effect would be seen in the ratio $\Delta m_{s} / \Delta m_{d}$, which could then be used to set the value of the SM expectation for $\left|V_{t d}\right|$. A $5 \%$ accuracy on $f_{B_{d}} \sqrt{B_{B}}$ would then probe a region of SUSY masses well beyond the reach of LEP2 and presumably Tevatron.

The range of masses $m(\tilde{t}) \lesssim m(t)$ is particularly interesting for another phenomenon which has connections to lattice calculations: this is baryogenesis at the EWK phase transition, a subject which was reviewed in this Conference by Fodor [26.

A second interesting example comes from tests of different speculations on the underlying structure of the quark mass matrix. Several models of quark mass matrices [27] predict for example the following relations:

$\left|\frac{V_{u b}}{V_{c b}}\right|=\sqrt{\frac{m_{u}}{m_{c}}} \quad\left|\frac{V_{t d}}{V_{t s}}\right|=\sqrt{\frac{m_{d}}{m_{s}}}$

Relations of this type emerge naturally, for example, in hierarchical $U(2)$ models for the flavour 


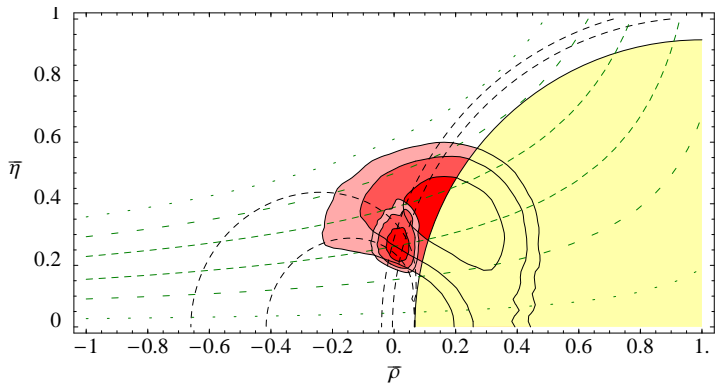

Figure 2. Confidence-level profiles for $\rho+i \eta$ [29]. The smaller regions incorporate the constraints given by the quark-mass relations in eqs. (23).

symmetry of quarks and leptons 28.

These relations can be used to predict $\rho, \eta$, or in turn can be tested against the extractions of $\rho, \eta$ from the data. Using as inputs:

$$
\begin{aligned}
Q & \equiv \frac{m_{s} / m_{d}}{\sqrt{1-\left(m_{u} / m_{d}\right)^{2}}}=22.7 \pm 0.8 \\
m_{u} / m_{d} & =0.553 \pm 0.043 \\
m_{c} / m_{s} & =8.23 \pm 1.5
\end{aligned}
$$

Barbieri, Hall and Romanino [29] obtained the results given in fig. 2 for $\rho+i \eta$. This determination of $\rho+i \eta$ is currently more precise than the SM fits. Improvements in the SM fits, and in the determination of the light quark masses, will allow stringent tests of these texture scenarios!

\section{3. $\epsilon^{\prime} / \epsilon$}

Two new results have appeared in 1999, from analyses of data subsets from $\mathrm{KTeV}$ [30] (FNAL) and NA48 [31] (CERN):

$$
\begin{array}{ll}
\operatorname{Re} \frac{\epsilon^{\prime}}{\epsilon}= & \\
\left(28.0 \pm 2.8_{\text {syst }} \pm 3.0_{\text {stat }}\right) \cdot 10^{-4} & \mathrm{KTeV} \\
\left(18.5 \pm 4.5_{\text {syst }} \pm 5.8_{\text {stat }}\right) \cdot 10^{-4} & \mathrm{NA} 48 \\
(21.2 \pm 2.8) \cdot 10^{-4} & \langle\text { World }\rangle
\end{array}
$$

These values of $\epsilon^{\prime} / \epsilon$ firmly establish the existence of direct $\mathrm{CP}$ violation in the Kaon system. These results correspond to a small fraction of the amount of data that will be analysed in the coming years:
- $\mathrm{KTeV}$ analysed only $25 \%$ of total data.

- NA48 analysed only $25 \%$ of available data. More data have been taken in 1999, and yet more will be collected in the year 2000 . The final sample size will be approximately 10 times larger than the presently analysed one.

- KLOE at the Frascati $\Phi$-factory has recently started taking data 32]. They expect to reach $\delta_{\text {stat }} \sim 10^{-3}$ within 1999 , and to start the dive towards the $10^{-4}$ sensitivity level in the close future.

- In all cases the leading contributions to $\delta_{\text {syst }}$ scale like $\delta_{\text {stat }}$.

- An accuracy of $\mathcal{O}\left(10^{-4}\right)$ is therefore expected overall within 2-3 years.

What about the theoretical predictions?

\subsection{Crude overview of $\left(\epsilon^{\prime} / \epsilon\right)_{\text {theory }}$}

The following discussion is based on the recent good reviews in refs. [22] and 33, 34.

To first approximation $\epsilon^{\prime} / \epsilon$ is dominated by the contribution of two operators:

$$
\begin{gathered}
\operatorname{Re} \frac{\epsilon^{\prime}}{\epsilon}=13 \operatorname{Im} \lambda_{t}\left[\frac{130 \mathrm{MeV}}{\mathrm{m}_{\mathrm{s}}\left(\mathrm{m}_{\mathrm{c}}\right)}\right]^{2}\left(\frac{\Lambda_{4}^{\overline{\mathrm{MS}}}}{340 \mathrm{MeV}}\right) \times \\
{\left[B_{6}^{(1 / 2)}\left(1-\Omega_{\eta+\eta^{\prime}}\right)-0.4 B_{8}^{(3 / 2)}\left(\frac{m_{t}}{165 \mathrm{GeV}}\right)^{2.5}\right]}
\end{gathered}
$$

where:

- $B_{6,8}$ are the bag parameters of the QCD and EWK penguin operators:

$$
\begin{aligned}
Q_{6} & =\left(\bar{s}_{\alpha} d_{\beta}\right)_{V-A} \sum_{q=u, d, s}\left(\bar{q}_{\beta} q_{\alpha}\right)_{V+A} \\
Q_{8} & =\left(\bar{s}_{\alpha} d_{\beta}\right)_{V-A} \sum_{q=u, d, s} e_{q}\left(\bar{q}_{\beta} q_{\alpha}\right)_{V+A}
\end{aligned}
$$

- $\operatorname{Im} \lambda_{t}=\operatorname{Im}\left[V_{t d} V_{t s}^{*}\right]=A^{2} \lambda^{5} \eta$

- $\Omega_{\eta+\eta^{\prime}} \sim 0.25$ is an $S U(3)$ breaking parameter. 


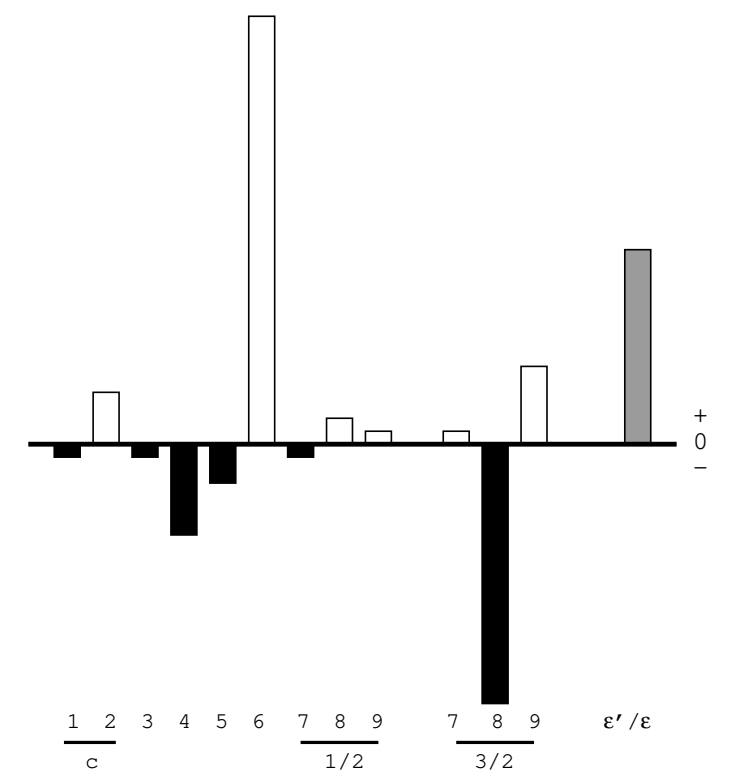

Figure 3. Breakdown of the contributions from all possible $\Delta S=14$-fermion operators, as estimated by the Rome group [35].

The contribution of the other $\Delta S=1$ operators is strongly suppressed, as can be seen from figs. 3 and 4 . Because of the possible large cancellation between the contributions of the two operators, the choice of input parameters is critical to determine the value and the uncertainty of the theoretical prediction for $\epsilon^{\prime} / \epsilon$.

In particular, the value of the non-perturbative matrix elements for the $Q_{6,8}$ operators is critical. Different approaches (lattice [36], 1/ $N$ expansion [37] and chiral quark model ( $\chi \mathrm{QM}$, 34] give results for $B_{8}^{(3 / 2)}$ consistent with the value of $0.8 \pm 0.2$. The situation for $B_{6}$ is much less clear. Pre-1998 lattice estimates [38] led to values in the range $0.9 \pm 0.3 . \chi \mathrm{QM}$ calculations 34] predict $B_{6}^{(1 / 2)}=1.4 \pm 0.4$, with a strong dependence on the value of the strange-quark mass. The $1 / N$ expansion [37] gives $B_{6}^{(1 / 2)}=1.6 \pm 0.1$, where large corrections due to $\mathcal{O}\left(p^{2} / N\right)$ subleading terms are included. More recent lattice estimates, based on staggered fermions [39] or domain wall fermions [40, give negative values for

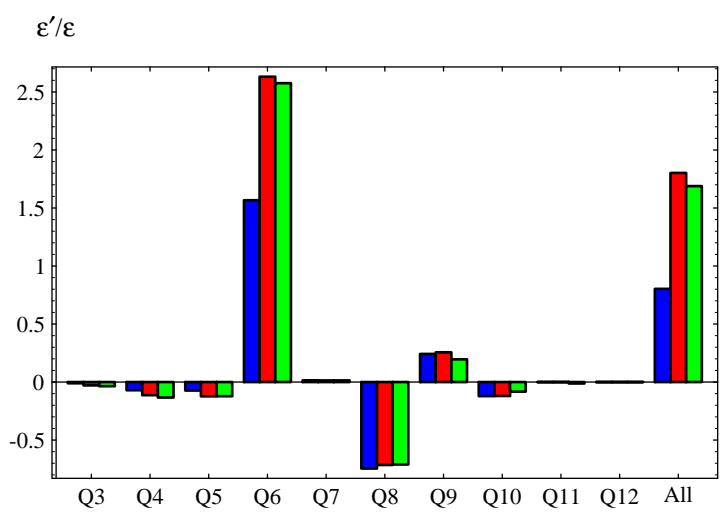

Figure 4. Breakdown of the contributions from all possible $\Delta S=14$-fermion operators, as estimated by the Trieste group in the $\chi \mathrm{QM}[34$. LO (black columns); chiral 1-loop (grey columns); full $\mathcal{O}\left(p^{4}\right)$ (light-grey).

$B_{6}$. In my modest opinion, these results just confirm the difficulty of the determination of $B_{6}$, and suggest that a rather large uncertainty should be attached to the input value used in the determination of $\epsilon^{\prime} / \epsilon$.

In ref. [22], the following parameter ranges have been proposed:

- $\operatorname{Im} \lambda=(1.33 \pm 0.14) \cdot 10^{-4}$, from CKM fit.

- $m_{s}{ }^{\left(m_{c}\right)}=130 \pm 25 \mathrm{MeV}$, mostly from Lattice, QCDSR.

- $\Lambda_{4}^{\overline{\mathrm{MS}}}=340 \pm 50 \mathrm{MeV}$, from $\alpha_{S}\left(M_{Z}\right)=$ $0.1185 \pm 0.003$.

- $B_{8}=0.8 \pm 0.2$

- $B_{6}=1 \pm 0.3$

Normalizing to the central values for the input parameters, and neglecting the $m_{t}$ dependence, one gets approximately:

$$
\begin{array}{r}
\operatorname{Re} \frac{\epsilon^{\prime}}{\epsilon}=7 \cdot 10^{-4} \times\left[1.9 B_{6}^{(1 / 2)}-B_{8}^{(3 / 2)}\right] \\
\times\left(\frac{\Lambda_{4}^{\frac{\epsilon S}{\mathrm{MS}}}}{340 \mathrm{MeV}}\right)\left(\frac{\operatorname{Im} \lambda_{t}}{1.34 \times 10^{-4}}\right)\left[\frac{130 \mathrm{MeV}}{\mathrm{m}_{\mathrm{s}}\left(\mathrm{m}_{\mathrm{c}}\right)}\right]^{2}
\end{array}
$$


which, for the central choices of the parameters, gives $\epsilon^{\prime} / \epsilon \sim 7 \cdot 10^{-4}$, far too small to agree with the data!

Taking a $10 \%$ shift in $\Lambda_{4}^{\overline{\mathrm{MS}}}, \operatorname{Im} \lambda$ and $m_{s}$ (consistent with a variations within $1 \sigma$ ), and using $\epsilon^{\prime} / \epsilon=2 \cdot 10^{-3}$, we get:

$$
\left[1.9 B_{6}^{(1 / 2)}-B_{8}^{(3 / 2)}\right] \sim 2
$$

With a value of $B_{8}$ consistent with the central theoretical value, 0.8 , one can solve this equation for $B_{6}=2$. Is this value really unacceptable to lattice?

The recent analysis of Bosch et al gives the following results, using different approaches to the description of the systematic uncertainties:

1. Gaussian errors for experimental inputs, flat for theory parameters (NDR):

$$
\frac{\epsilon^{\prime}}{\epsilon}=\left(7.7_{-3.5}^{+6.0}\right) \cdot 10^{-4}
$$

2. Range covered by the scan of parameters (NDR):

$$
\frac{\epsilon^{\prime}}{\epsilon}=(1.05 \rightarrow 28.8) \cdot 10^{-4}
$$

Similar results have been obtained in the recent analysis of the Rome group [23], which also contains an interesting appraisal of the overall uncertainties coming from the evaluation of the nonperturbative matrix elements, and of the correlation between the various inputs used in the theoretical calculations.

\subsection{New physics in $\epsilon^{\prime} / \epsilon$ ?}

Because of the large uncertainty due to the evaluation of $B_{6}$, it is clearly premature to draw conclusions on the possible presence of new physics from the measurement of $\epsilon^{\prime} / \epsilon$. Nevertheless, it is worth mentioning that several papers have already appeared, trying to explore how much room is available for contributions beyond the SM. In the case of supersymmetry, one needs to resort to extensions of the minimal scenarios, in particular to models with explicit $\not \mathrm{P}$ phases in the squark mass matrices. Possible contributions to $\epsilon^{\prime} / \epsilon$ then emerge, proportional to $\operatorname{Im}\left(M_{12}^{2}\right)_{L R}$, where $M_{12}^{2}$ is a contribution to the scalar quark mass matrix mixing left and right scalars of the first two generations. In these models, contributions are also expected to the neutron electric dipole moment $\left(d_{n}^{S U S Y} \propto \operatorname{Im}\left(M_{q q}^{2}\right)_{L R}\right)$. Current experimental constraints $\left(<0.94 \cdot 10^{-25} e \mathrm{~cm}\right)$ make a possible contribution to $\epsilon^{\prime} / \epsilon$ of order $10^{-3}$ unnatural, although still possible [41].

Other scenarios involve anomalous $Z d \bar{s}$ vertices 42]. In this case, anomalies in the rare Kaon decays $B R\left(K^{+} \rightarrow \pi^{+} \nu \bar{\nu}\right)$ and $B R\left(K_{L} \rightarrow \pi^{0} \nu \bar{\nu}\right)$ should appear [43], which will be testable in the experiments planned for the near future (KAMI at FNAL, E949 at BNL).

\section{HIGH ENERGY JETS AND QCD}

While it is clear that the contribution to flavour physics is the most fundamental one that lattice QCD can give at this time to HEP phenomenology, there are other areas where progress is limited by a poor understanding of the nonperturbative phase of QCD. Among these, we list for example: hadronic structure functions, power corrections to high- $Q^{2}$ phenomena, definition of the top quark mass, etc. I will illustrate with examples the relevance of some of these points. It is not clear to me whether lattice QCD can already contribute significantly to these problems, but I understand that progress is in sight.

\subsection{Jet production at the Tevatron}

At the Tevatron, jets up to $450 \mathrm{GeV}$ transverse momentum have been observed 44,45. These data can be used for many interesting purposes:

- Tests of QCD: calculations are available up to NLO 46.

- Extract information on the partonic densities, $f_{q, g}\left(x, Q^{2}\right)$ at large $Q^{2}$.

- Look for deviations from QCD (e.g. resonances in the dijet mass spectrum), explore quark structure at small distances.

The accessible range of transverse energies corresponds to values of $x \gtrsim 0.5$, at $Q^{2} \simeq 160,000$ $\mathrm{GeV}^{2}$. This is a domain of $x$ and $Q^{2}$ not accessible to DIS experiments, such as those running 


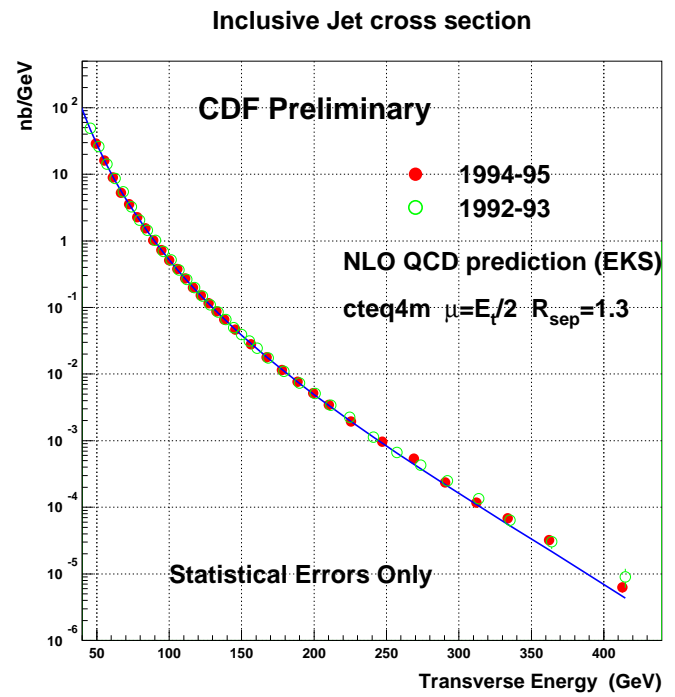

Figure 5. Inclusive jet transverse energy $\left(E_{T}\right)$ distribution as measured by CDF, compared to the absolute NLO QCD calculation.

at HERA. The current agreement between theory and data is at the level of $30 \%$ over 8 orders of magnitude of cross-section, from $E_{T} \sim 20$ to $E_{T} \sim 450 \mathrm{GeV}$ (see fig. 5) In spite of the general good agreement, a large dependence on the chosen set of parton densities 47,48] is present, as shown in fig. 6. The presence of this uncertainty limits the use of high- $E_{T}$ jet data to set constraints on possible new physics.

An important question is therefore the following: to which extent do independent measurements of parton densities constrain the knowledge of PDFs at large- $x$, and what is the residual uncertainty on the jet $E_{T}$ distributions?

To address this issue, let us first show what is the relative contribution of different initial state partons to the jet cross section. This is plotted in fig. 7, where some standard PDF set (CTEQ4M 48 in this case) was chosen. At the largest energies accessible to today's Tevatron data, $80 \%$ of the jets are produced by collisions involving only initial state quarks. The remaining $20 \%$ comes from processes where at least one

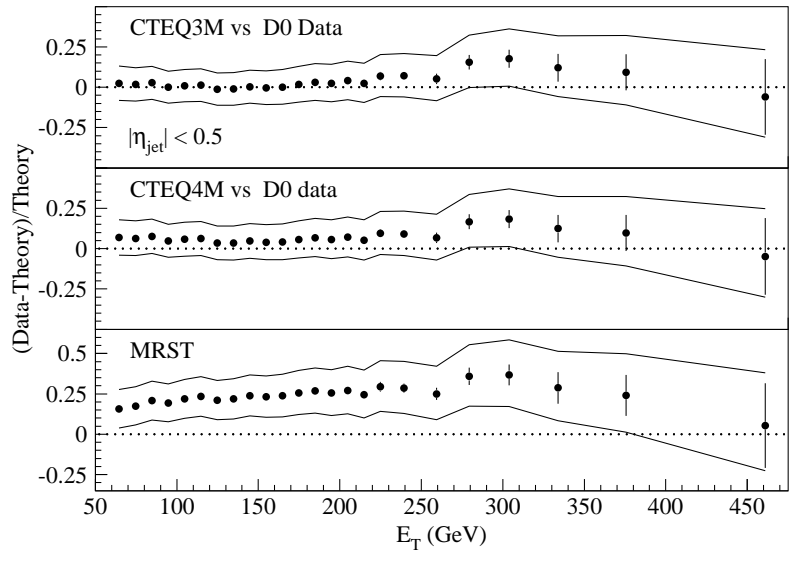

Figure 6. Deviations of QCD predictions from D0 jet data for various sets of PDFs.

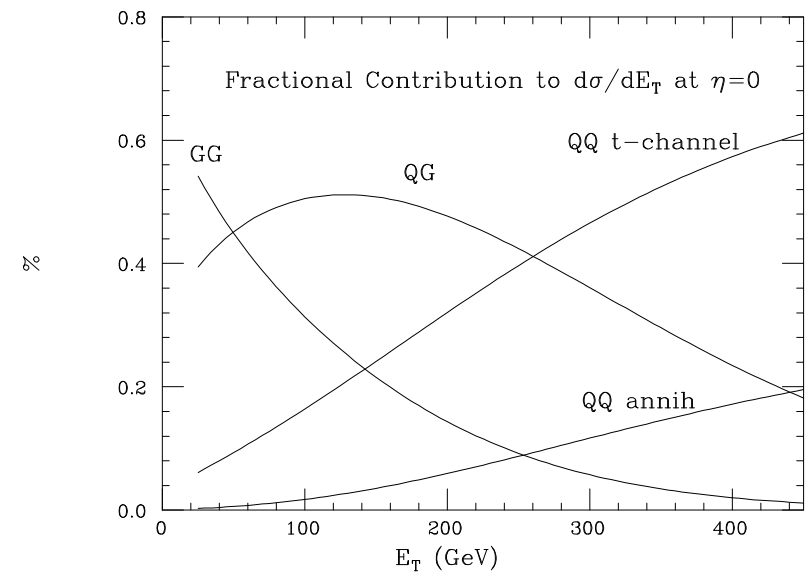

Figure 7. Contributions from different initial states to the jet cross section at $\sqrt{s}=1.8 \mathrm{TeV}$ 
gluon was present in the initial state.

Quark densities at large- $x$ quarks are constrained by DIS data to within few percent, leading to an overall uncertainty on the high- $E_{T}$ jet rate of at most $5 \%$. What is the uncertainty on the remaining $20 \%$ coming from gluon-induced processes? How are we guaranteed that the gluons are known to better than a factor of 2 , limiting the overall uncertainty to $20-30 \%$ ?

The only independent constraint on $f_{g}\left(x, Q^{2}\right)$ comes from fixed-target production of prompt photons. This process is induced at LO by two mechanisms, $q \bar{q} \rightarrow g \gamma$ and $q g \rightarrow q \gamma$. In $p N$ collisions $g(x) \gg \bar{q}(x)$, and therefore

$\frac{d \sigma}{d E_{T}}(q g \rightarrow q \gamma) \gg \frac{d \sigma}{d E_{T}}(q \bar{q} \rightarrow g \gamma)$

Data from FNAL and CERN fixed target experiments can therefore be used to extract $f_{g}\left(x, Q^{2}\right)$ at large $x$. Unfortunately, a comparison of data and NLO theory shows inconsistencies at small $E_{T}$ between the various experiments, as shown in fig. 8 49. As a possible explanation for these discrepancies, the presence of a large non-perturbative contribution from the intrinsic $k_{T}$ of partons inside the nucleon has been suggested 50,51]. This gives rise to power-like corrections to the spectrum of order $k_{T} / p_{T}$, with possibly very large coefficients due to to the steepness of the spectrum itself. The effect of the intrinsic $k_{T}$ is to smear the $p_{T}$ distribution, as shown in fig. 9. Inclusion of these effects, however, has a big impact also on the rate at large $E_{T}$ (i.e. $x \sim 0.6$ ). Due to the large size of the effects, and to their intrinsic non-perturbative nature (which means that they cannot be understood from first principles, and need to be described by ad hoc models), it is hard to trust the theoretical predictions obtained in this way, and to claim that prompt photons provide a reliable way of extracting the gluon content of the proton at large $x$. Recent theoretical improvements, such as the resummation of large- $x_{T}$ logarithms 52 54, should help understanding the large- $x$ problem, but more work is necessary. In conclusion, the issue of the large- $x$ behaviour of $f_{g}(x)$ is still an open problem.

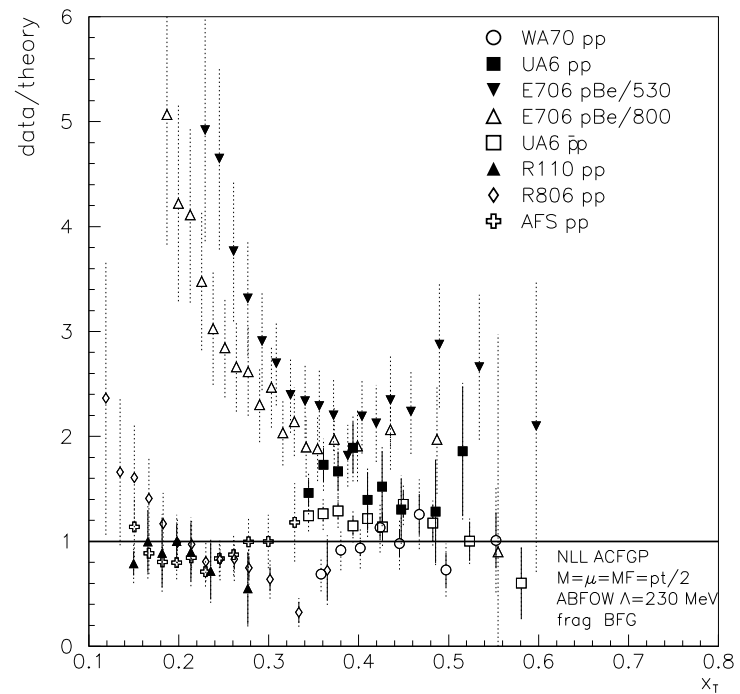

Figure 8. Relative deviations between NLO QCD and prompt photon data, as a function of $x_{T}=2 p_{T} / \sqrt{S}$, for various fixed target experiments.

Concerning the possible eccess observed by $\mathrm{CDF}$ in its highest $E_{T}$ jet data 44], additional input will be available with the data from the upcoming run of the Tevatron (due to start in the Summer 2000), thanks to an increased energy ( $\sqrt{S} \rightarrow 2 \mathrm{TeV}, 10 \%$ increase). Should the eccess be due to a problem with the gluon density at large $x$, a discrepancy similar to the one observed at $1.8 \mathrm{TeV}$ will appear at jet $E_{T}$ values $10 \%$ larger. If the eccess is instead due to really new phenomena, one expects the excess to appear at the same value of $E_{T}$ as seen in the data at $1.8 \mathrm{TeV}$. Time will tell!

Needless to say, any theoretical input on the expected behaviour of the gluon density at large $x$, and on the precise way in which non-perturbative effects such as the intrinsic motion of quarks and gluons inside the proton are to be implemented in perturbative calculations, would be of great interest. This is an interesting challange to the lattice community! 


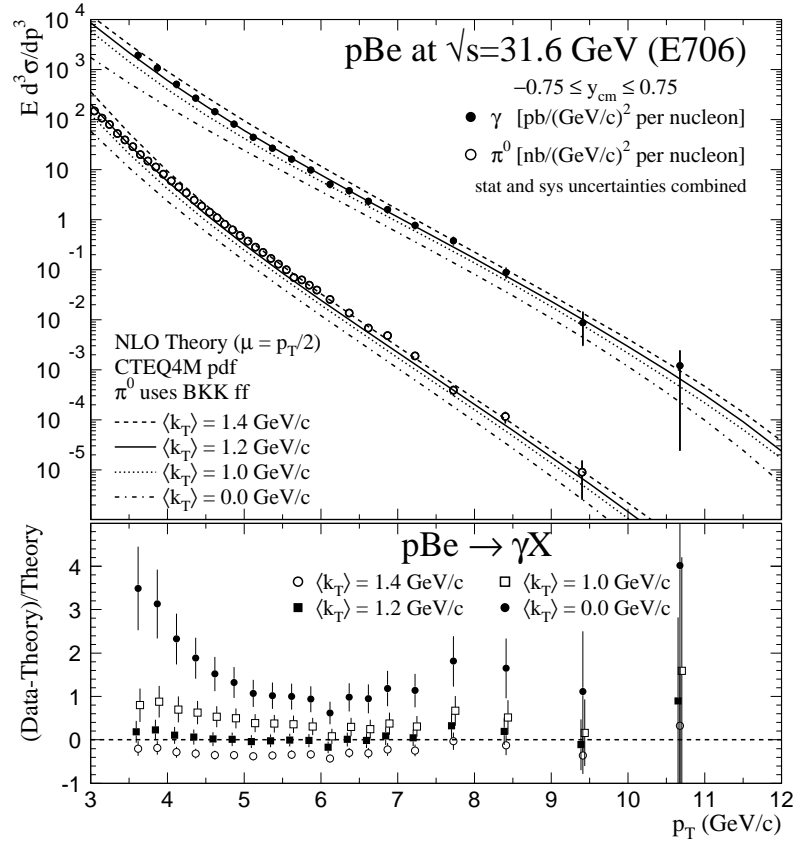

Figure 9. Comparison of E706 data 50 with NLO QCD, before and after inclusion of an intrinsic- $k_{T}$.

\section{REFERENCES}

1. C. Caso et al., Review of Particle Properties, Eur. Phys. J. C3 (1998), 1.

2. M. Neubert, Int. J. Mod. Phys. A11, 4173 (1996) hep-ph/9604412. I. Bigi, M. Shifman and N. Uraltsev, Ann. Rev. Nucl. Part. Sci. 47, 591 (1997) hep-ph/9703290.

3. D. Abbaneo et al., internal note of the LEP heavy-flavour steering group, LEPHFS 99-02, available from http://home.cern.ch/r/roudeau/www/ bc_steering.html.

4. D. Abbaneo et al., internal note of the LEP heavy-flavour $V_{c b}$ working group, submitted to the 1999 EPS Conference, Tampere, 15-21 July 1999, available from http://www.cern.ch/LEPVCB/

5. I.I. Bigi, hep-ph/9907270.

6. S. Hashimoto et al., hep-ph/9906376.

7. M. Artuso, plenary talk at the 1999 EPS Conference, Tampere, 15-21 July 1999.

8. S. Hashimoto, hep-lat/9909136.

9. B.H. Behrens et al. [CLEO Collab.], hepex/9905056.

10. I. Bigi, R.D. Dikeman and N. Uraltsev, Eur. Phys. J. C4, 453 (1998) hep-ph/9706520. N. Uraltsev, hep-ph/9905520. A.H. Hoang, Z.Ligeti and A.V. Manohar, Phys. Rev. Lett. 82, 277 (1999); Phys. Rev. D59, 074017 (1999).

11. K. Melnikov and A. Yelkhovsky, Phys. Rev. D59, 114009 (1999) hep-ph/9805270. A.H. Hoang, hep-ph/9905550. M. Beneke and A. Signer, hep-ph/9906475.

12. V. Gimenez, L. Giusti, F. Rapuano and G. Martinelli, hep-lat/9909138.

13. D. Abbaneo et al., internal note of the LEP heavy-flavour $V_{u b}$ working group, LEPVUB 99-01, available from http://home.cern.ch/battagl/vub/vub.html.

14. F. De Fazio and M. Neubert, JHEP 06, 017 (1999) hep-ph/9905351.

15. P.F. Harrison and H.R. Quinn [BABAR Collab.], "The BaBar physics book: Physics at an asymmetric B factory,"

16. The LEP/SLD/CDF B oscillations working group, prepared for the 1999 
Summer Conferences, available through http://www.cern.ch/LEPBOSC/.

17. S.R. Sharpe, hep-lat/9811006.

18. F. Parodi, P. Roudeau and A. Stocchi, hep$\mathrm{ex} / 9903063$.

19. V. Giménez and G. Martinelli, Phys. Lett. B398, 135 (1997)

20. S. Mele, Phys. Rev. D59, 113011 (1999) hep$\mathrm{ph} / 9810333$.

21. A. Ali and D. London, Eur. Phys. J. C9, 687 (1999) hep-ph/9903535.

22. S. Bosch, A.J. Buras, M. Gorbahn, S. Jager, M. Jamin, M.E. Lautenbacher and L. Silvestrini, hep-ph/9904408.

23. M. Ciuchini, E. Franco, L. Giusti, V. Lubicz and G. Martinelli, hep-ph/9910236.

24. T. Affolder et al. [CDF Collab.], hepex/9909003.

25. A. Brignole, F. Feruglio and F. Zwirner, Z. Phys. C71, 679 (1996) hep-ph/9601293.

S. Bertolini, F. Borzumati, A. Masiero and G. Ridolfi, Nucl. Phys. B353, 591 (1991).

26. Z. Fodor, in these proceedings.

27. H. Fritzsch, Nucl. Phys. B155, 189 (1979).

28. A. Pomarol and D. Tommasini, Nucl. Phys. B466, 3 (1996) hep-ph/9507462. R. Barbieri, G. Dvali and L.J. Hall, Phys. Lett. B377, 76 (1996) hep-ph/9512388.

29. R. Barbieri, L.J. Hall and A. Romanino, Nucl. Phys. B551, 93 (1999) hep-ph/9812384.

30. A. Alavi-Harati et al. [KTeV Collab.], Phys. Rev. Lett. 83, 22 (1999) hep-ex/9905060.

31. V. Fanti et al. [NA48 Collab.], hepex/9909022.

32. S. Bertolucci, [Kloe Collab.], presented at the 1999 Lepton-Photon Symposium,

33. A.J. Buras, hep-ph/9905437.

34. S. Bertolini, M. Fabbrichesi and J.O. Eeg, hep-ph/9802405.

35. M. Ciuchini, E. Franco, G. Martinelli, L. Reina and L. Silvestrini, Z. Phys. C68, 239 (1995) hep-ph/9501265.

36. R. Gupta, Nucl. Phys. Proc. Suppl. 63, 278 (1998) hep-lat/9710090.

37. T. Hambye, G.O. Kohler, E.A. Paschos, P.H. Soldan and W.A. Bardeen, Phys. Rev. D58, 014017 (1998) hep-ph/9802300. T. Hambye, G.O. Kohler, E.A. Paschos and
P.H. Soldan, hep-ph/9906434.

38. G. Kilcup, Nucl. Phys. Proc. Suppl. 20, 417 (1991). S.R. Sharpe, Nucl. Phys. Proc. Suppl. 20, 429 (1991). D. Pekurovsky and G. Kilcup, Nucl. Phys. Proc. Suppl. 63, 293 (1998) heplat/9709146.

39. D. Pekurovsky and G. Kilcup, heplat/9812019.

40. T. Blum et al., hep-lat/9908025.

41. A. Masiero and H. Murayama, Phys. Rev. Lett. 83, 907 (1999) hep-ph/9903363. R. Barbieri, R. Contino and A. Strumia, hep$\mathrm{ph} / 9908255$.

42. G. Colangelo and G. Isidori, JHEP 09, 009 (1998) hep-ph/9808487.

43. A.J. Buras, G. Colangelo, G. Isidori, A. Romanino and L. Silvestrini, hep-ph/9908371. G. Colangelo, G. Isidori and J. Portoles, hep$\mathrm{ph} / 9908415$.

44. F. Abe et al., CDF Collab., Phys. Rev. Lett. 77 (1996) 438.

45. B. Abbott et al., D $\emptyset$ Collab., hep-ex/9807018.

46. F. Aversa, P. Chiappetta, M. Greco and J.Ph. Guillet, Nucl. Phys. 327 (1989) 105; S.D. Ellis, Z. Kunszt and D.E. Soper, Phys. Rev. Lett. 64, 2121 (1990);

W.T. Giele, E.W. Glover and D.A. Kosower, Nucl. Phys. B403, 633 (1993) hep$\mathrm{ph} / 9302225$.

47. A.D. Martin, R.G. Roberts, W.J. Stirling and R.S. Thorne, Eur. Phys. J. C4, 463 (1998) hep-ph/9803445.

48. H.L. Lai et al. [CTEQ Collab.], hep$\mathrm{ph} / 9903282$.

49. P. Aurenche, et al., hep-ph/9811382

50. L. Apanasevich et al., E706 Collab., Phys. Rev. Lett. 81 (1998) 2642.

51. L. Apanasevich et al., Phys. Rev. 59 (1999) 074007.

52. N. Kidonakis and G. Sterman, Nucl. Phys. B505, 321 (1997) hep-ph/9705234.

53. E. Laenen, G. Oderda and G. Sterman, Phys. Lett. B438, 173 (1998) hep-ph/9806467.

54. S. Catani, M.L. Mangano and P. Nason, JHEP 07, 024 (1998) hep-ph/9806484:

S. Catani, M.L. Mangano, P. Nason, C. Oleari and W. Vogelsang, JHEP 03, 025 (1999) hep- 
ph/9903436. 